\section{Commentary: Low cardiac output syndrome: A definition or a diagnosis code?}

\author{
Bryan A. Whitson, MD, PhD
}

Since the advent of cardiothoracic surgery, the patients and their perioperative management has been complex. As we have gained a deeper understanding of the physiology, the perturbation of that physiology with our surgical and critical care interventions, and our expanding array of monitoring and treatment modalities, our understanding of the management of the patients has evolved as well. In the past, a low blood pressure or cardiac output after surgery has gone by many names-hypotension, cardiogenic shock, vasoplegia, heart failure, stunning, and distributive shock, to name a few. Today, we have low cardiac output syndrome (LCOS). Although there are different definitions, it in general it is hypotension or "shock" in a patient after cardiac surgery.

From some perspectives, this is a nebulous International Statistical Classification of Diseases and Related Health Problems, Tenth Revision, billing code that would track to heart failure, unspecified (or not otherwise specified), which would facilitate the critical care management and billing in the perioperative period by intensive care physicians. From other perspectives, it is a significant entity in of itself that reflects a patient with significant derangements in their cardiovascular system in need of vasopressor or inotropic support. The presence of LCOS may reflect a sicker patient and may portend a worse outcome.

In this issue of the Journal, the group from Cleveland Clinic Foundation lead by Dr Andra Duncan and

From the Division of Cardiac Surgery, Department of Surgery, The Ohio State University Wexner Medical Center, Columbus, Ohio.

Disclosures: B.A.W. is a consultant/speaker for Abbott Laboratories, is a proctor for Medtronic, and serves on the Clinical Events Committee of TransMedics OCS.

The Journal policy requires editors and reviewers to disclose conflicts of interest and to decline handling or reviewing manuscripts for which they may have a conflict of interest. The editors and reviewers of this article have no conflicts of interest.

Received for publication Sept 10, 2020; revisions received Sept 10, 2020; accepted for publication Sept 11, 2020; available ahead of print Sept 14, 2020.

Address for reprints: Bryan A. Whitson, MD, PhD, Division of Cardiac Surgery, Department of Surgery, Ohio State University Medical Center, N-816 Doan Hall, 410 W. 10th Ave, Columbus, OH 43210 (E-mail: bryan.whitson@osumc.edu).

J Thorac Cardiovasc Surg 2022;163:1902-3 0022-5223/ $\$ 36.00$

Copyright $₫ 2020$ Published by Elsevier Inc. on behalf of The American Association for Thoracic Surgery

https://doi.org/10.1016/j.jtcvs.2020.09.040

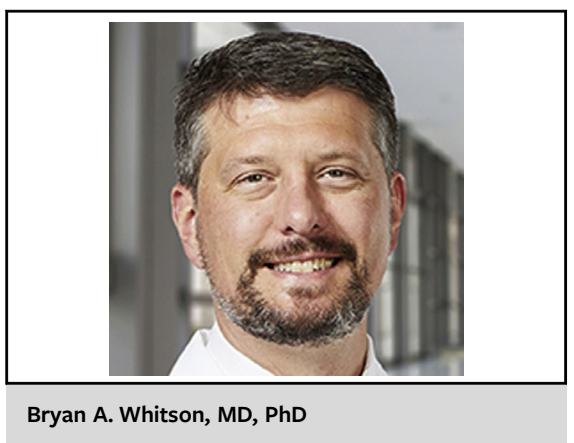

CENTRAL MESSAGE

There is a role for deeper clinical understanding resulting in new diagnoses and conditions, although this expansion needs to be balanced by clear definitions.

colleagues uses a large administrative database to elucidate the impact of LCOS in terms of cost and resource use and predictive risk factors. ${ }^{1}$ Duncan and colleagues used the Premier Healthcare Database, evaluating 164 hospitals and 59,810 patients who underwent cardiac surgery over a 2 -year period. Although bound by the inherent limitations and bias of using a retrospective, large, multi-institutional databases, the authors attempted to employ a concrete definition of LCOS: (1) need for mechanical circulatory support with intra-aortic balloon pump, left ventricular assist device, or extracorporeal membrane oxygenation during or after surgery, and/or (2) hemodynamic instability requiring continued pharmacologic support with $\geq 2$ inotropic medications on postoperative day 1 . These criteria were identified by procedure codes and charges records. This potentially is more objective than diagnoses codes, which may be vague, cover diverse real pathologies, and are difficult to validate.

Nevertheless, Duncan and colleagues effectively use the power of such a large dataset and the detail provide within the Premier data registry to determine that postoperative LCOS is associated with greater cost, longer intensive care unit length of stay, greater readmission rates, and greater in-hospital mortalities. Irrespective of the nuances of the definition, the presence of LCOS has a significant impact on our patients and their outcomes. Striving to use a standard definition, as done in the current study, is important in allowing us objective criteria in identification as well as comparing study to study 
and intervention to intervention to identify therapeutic approaches that will meaningfully impact our patients in a positive manner.

\section{Reference}

1. Duncan AE, Kartashov A, Robinson S, Randall D, Zhang K, Luber J, et al. Risk factors, resource use, and cost of postoperative low cardiac output syndrome. J Thorac Cardiovasc Surg. 2022;163:1890-8.e10. 